\title{
Sistema de Localización y Posicionamiento de Piezas utilizando Visión Artificial
}

\author{
Javier de Lope, Francisco Serradilla, José G. Zato
}

\begin{abstract}
Resumen
En el presente artículo describimos un sistema automatizado para reconocimiento, localización y posicionamiento de piezas de fabricación para la industria aeronáutica. Dichas piezas, compuestas de un material flexible, son depositadas de forma automática en una mesa de trabajo de $2 \times 2 \mathrm{~m}^{2}$ provenientes de un proceso de automatización anterior. La tarea a desarrollar por el sistema consiste en una primera identificación y validación de la pieza para, posteriormente, encontrar su localización exacta y conducirla a una posición y orientación final gracias a un robot de tipo pórtico. Las especificaciones del sistema indican que el error de $\mathrm{mm}$. Para alcanzar la precisión requerida, el sistema dispone de dos

cámaras de visión artificial; la primera obtiene una panorámica general, y la segunda, moviéndose conjuntamente con el efector del pórtico, permite tomar una imagen muy próxima al objeto y por tanto mucho más precisa. Esta arquitectura doble permite abaratar considerablemente el hardware del sistema gracias al uso
\end{abstract}

\section{Introducción}

Dentro de los procesos de fabricación de material aeronáutico existe una fase denominada conformado de patrones, cuyo objetivo es reforzar mediante un recubrimiento diversas partes de las aeronaves. Estos patrones están compuestos de fibra de carbono, fibra de vidrio o de kevlar. La mecanización del proceso de conformado consta de tres etapas: el cortado del patrón, su colocación en el útil de forrado, y el forrado definitivo de la pieza. Una vez cortado el patrón se deposita en una mesa de trabajo, donde deberá ser reconocido y posicionado en una zona determinada para su colocación en el útil.

Este artículo se centra en el diseño e implementación de un sistema software que, utilizando técnicas de visión artificial, realice las tareas de reconocimiento, localización y posicionamiento de los patrones sobre la mesa de trabajo. Como requisito inicial se cuenta con que el error de posicionamiento en las piezas debe ser menor que $\pm 0.5 \mathrm{~mm}$ en posición y $\pm 0.5^{\circ}$ en orientación. A tal efecto, hemos utilizado los siguientes elementos:

- Un robot pórtico con cuatro grados de libertad (tres de traslación en los ejes X, Y, Z y uno de rotación) con su correspondiente controlador que opera sobre la mesa de trabajo de dimensiones de $2 \times 2 \mathrm{~m}^{2}$.

- Dos cámaras de visión CCD Sony conectadas a una tarjeta digitalizadora Matrox IP-8. Una de las cámaras se utiliza para tomar imágenes lejanas de la zona de trabajo completa. La otra, solidaria con el efector del robot, se utiliza para tomar imágenes cercanas de una región de la pieza y refinar las

- Un ordenador 486, encargado de procesar las imágenes y enviar las instrucciones de posicionamiento al controlador del robot.

Para cumplir los objetivos definidos, el sistema software se ha articulado en una serie de fases o etapas que se enumeran a continuación:

Preproceso y segmentación. Dada una imagen en 256 niveles de gris debemos ser capaces de obtener una imagen binaria, donde los puntos pertenecientes a la pieza y los puntos del fondo se etiquetarán con diferentes valores.

Reconocimiento y validación. Utilizando la imagen tomada con la cámara lejana, previamente segmentada, debemos reconocer el tipo de pieza que se encuentra sobre la superficie de trabajo. Una vez efectuada 
- La cámara adosada al robot pórtico se envía a la posición del vértice de referencia. En este punto se toma una nueva imagen cercana de la pieza con la que se puede determinar la posición del vértice de referencia con el error requerido.

Posicionamiento. Utilizando el modelo geométrico de la pieza se calcula su posición, referida al centro de masas, y el movimiento (traslación y rotación) necesario para depositar la pieza en su punto de destino. Seguidamente se envían las instrucciones apropiadas al controlador del robot.

Una restricción adicional del sistema es que debe ser capaz de funcionar en tiempo real. Por ello, los algoritmos encargados de llevar a cabo las diferentes tareas deben ser cuidadosamente seleccionados.

\section{Caracterización Geométrica}

Para manejar de forma conveniente la información aportada por las cámaras e integrarla en comandos de movimiento al brazo mecánico, se ha efectuado una caracterización geométrica del entorno de operación mediante la definición de una serie de sistemas de referencia que incluye los asociados a las cámaras lejana $\mathrm{R}_{\mathrm{L}}=\left\{\mathrm{O}_{\mathrm{L}} ; \mathrm{x}_{\mathrm{L}}, \mathrm{y}_{\mathrm{L}}, \mathrm{z}_{\mathrm{L}}\right\}$ y cercana $\mathrm{R}_{\mathrm{C}}=\left\{\mathrm{O}_{\mathrm{C}} ; \mathrm{x}_{\mathrm{C}}, \mathrm{y}_{\mathrm{C}}, \mathrm{z}_{\mathrm{C}}\right\}$. Un esquema de esta caracterización se muestra en la Figura 1.

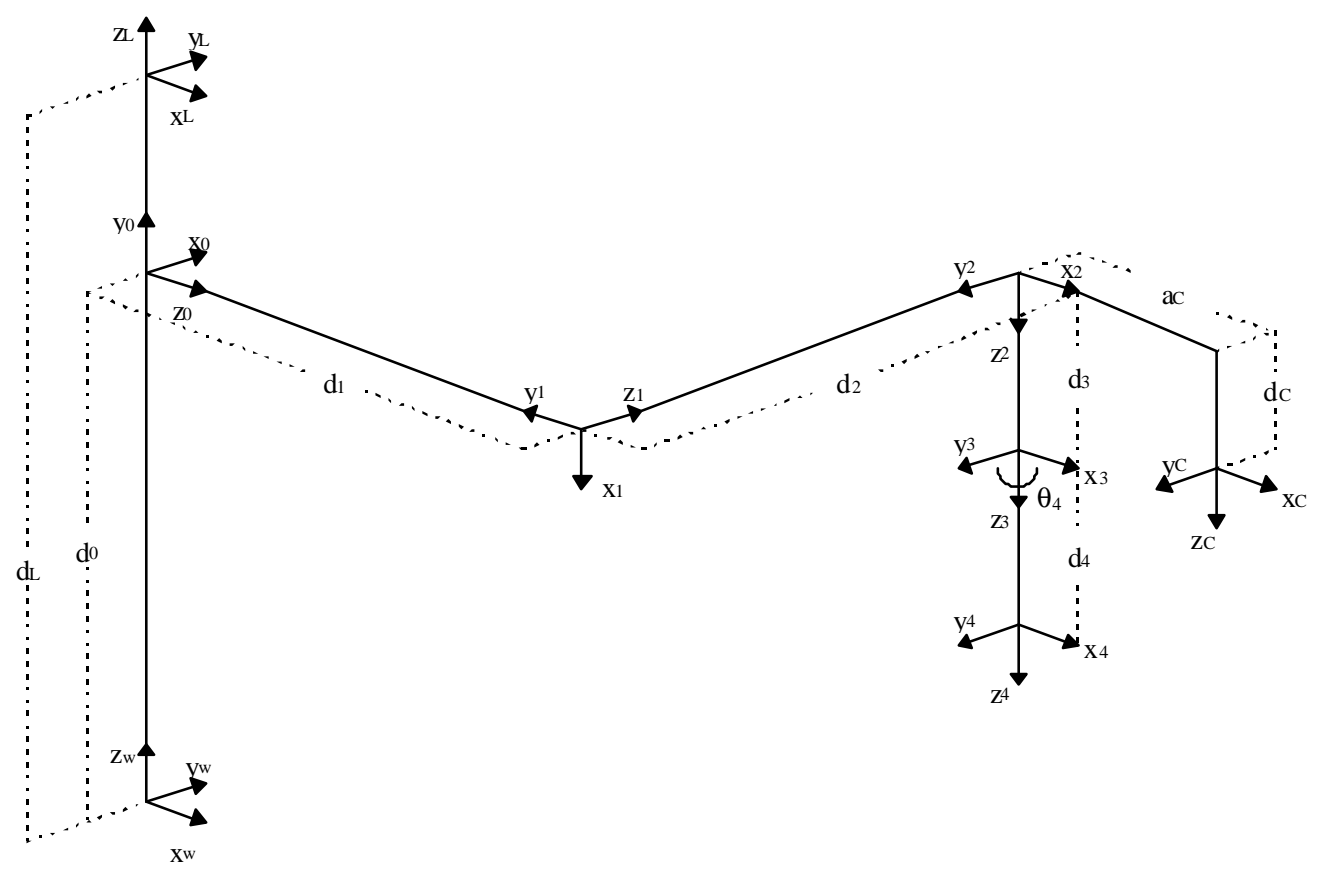

Figura 1: Esquema geométrico del entorno de operación

Utilizando los mecanismos habituales, se han obtenido los parámetros de Denavit y Hartenberg [Denavit, 55] que permitirán definir las expresiones de cambio de sistemas de referencia, que se relacionan en la Tabla 1. En la tabla se muestran entre paréntesis los parámetros que se dejan libres para calibrar algunas de las partes de los elementos mecánicos. En concreto, $\mathrm{d}_{0}$ es la altura a la que se sitúa el pórtico del sistema de referencia base o del mundo, $\mathrm{d}_{4}$ es la longitud del efector, $\mathrm{a}_{\mathrm{C}} \mathrm{y} \mathrm{d}_{\mathrm{C}}$ son las distancias de separación y altura de la cámara cercana desde el referencial final del segundo segmento del manipulador, respectivamente, y $d_{\mathrm{L}}$ es la altura a la que está colocada la cámara lejana desde el sistema de referencia del 
${ }_{1}, \mathrm{~d}_{2}, \mathrm{~d}_{3}$ y $\theta_{4}$ se dejan variables para expresar las coordenadas articulares del robot y definir la posición y orientación del efector y de la cámara cercana con respecto del sistema de referencia del mundo. Las expresiones matriciales (siguiendo la notación de [Schilling, 90]) que expresan estos cambios de referenciales se muestran a continuación:

$$
\begin{gathered}
T_{\text {Base }}^{\text {Efector }}=\left(\begin{array}{cccc}
\cos \Theta_{4} & -\operatorname{sen} \theta_{4} & 0 & d_{1} \\
-\operatorname{sen} \theta_{4} & -\cos \Theta_{4} & 0 & d_{2} \\
0 & 0 & -1 & d_{0}-d_{3}-d_{4} \\
0 & 0 & 0 & 1
\end{array}\right) \\
T_{\text {Base }}^{\text {Cercana }}=\left(\begin{array}{cccc}
1 & 0 & 0 & d_{1}+a_{c} \\
0 & -1 & 0 & d_{2} \\
0 & 0 & -1 & d_{0}-d_{c} \\
0 & 0 & 0 & 1
\end{array}\right)
\end{gathered}
$$

\section{Preproceso y Segmentación}

Antes de poder operar con la imagen de la pieza, deben ser discriminados los pixels correspondientes a la pieza de los del fondo. En nuestro problema estas tareas no son excesivamente complejas, debido a que las piezas son de color negro y la mesa de trabajo de color blanco. Este hecho produce diferencias acusadas entre los puntos de la imagen que corresponden a la pieza y los que corresponden a la mesa. Por tanto no es necesario ningún tipo de preproceso y la segmentación puede realizarse umbralizando la imagen original —en 256 niveles de gris - con un umbral fijo establecido en 128. A los puntos con valores de intensidad por encima o iguales a 128 asignaremos un valor de 255 (pieza) y a los puntos por debajo de 128 asignaremos un valor de 0 (fondo).

El único problema encontrado en esta fase surgió del hecho de que las piezas presentaban un tramado de agujeros apreciable en las imágenes captadas por la cámara cercana. Este problema se resolvió utilizando técnicas de morfología matemática, realizando un cierre de la imagen [Dougherty, 92].

\section{Reconocimiento y Validación}

Aunque las especificaciones del proyecto solicitaban solamente efectuar una validación de la pieza en términos de aceptación o rechazo, dependiendo de si la pieza era o no la esperada, esta validación requería en sí misma una identificación previa y, posteriormente, una comprobación. Desde este punto de vista, el problema puede abordarse desde la Teoría de Reconocimiento de Formas. 


\subsection{Piezas Prototipo y Piezas Percibidas}

Según la Teoría de Reconocimiento de Formas, cada pieza que llega al sistema pertenece a alguna de las posibles clases o patrones de un universo de trabajo. Cada clase va a estar representada por un prototipo, que en nuestro caso se construirá a partir del modelo geométrico de cada tipo de pieza. Tanto los prototipos como las piezas reales percibidas se describen a través de un conjunto de valores numéricos llamados , que usualmente se obtienen a partir de las imágenes tomadas con la cámara. Estos valores se agrupan en un vector, llamado vector de características. Como aportación original, en nuestro proyecto extraemos el vector de características de los prototipos a partir del modelo geométrico de la pieza. En la Figura 2 se muestra el esquema general de un reconocedor de formas.

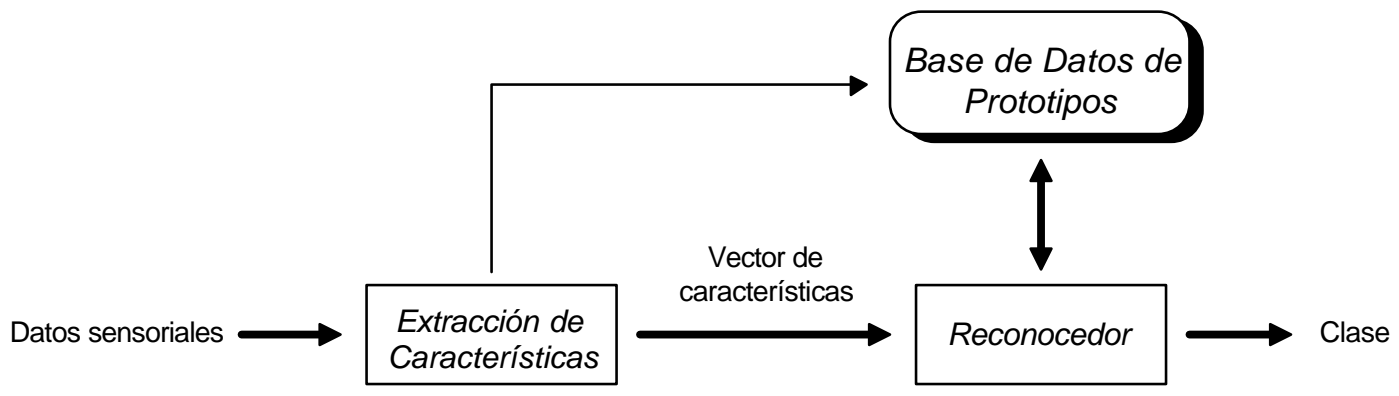

Figura 2: Esquema general de un reconocedor de formas

Tenemos, por tanto, dos clases o categorías de piezas:

- Piezas prototipo: capturan la esencia de todas las piezas pertenecientes a una misma clase. La práctica habitual es extraer el vector de características del prototipo de cada clase promediando los vectores de un gran número de ocurrencias de individuos de dicha clase. En nuestro problema, sin embargo, esta esencia se encuentra recogida en el modelo geométrico de la pieza, por ello el vector de características se extraerá a partir de este modelo. En primer lugar se dibujará la pieza en la pantalla, y a partir de esta imagen calcularemos los valores para las características. Algunas características, no obstante, se calcularán directamente a partir del modelo.

- Piezas percibidas : responden a ocurrencias de una clase, es decir, son piezas reales captadas por una cámara. El vector de características que describe a una pieza percibida se obtiene a partir de la imagen captada, previamente segmentada.

El sistema dispone de una base de datos que contiene el modelo geométrico de cada pieza prototipo. En la primera versión del sistema la geometría de las piezas está restringida a cuadriláteros. Según esto, su geometría puede describirse suministrando los cuatro vértices del modelo. A partir de ellos el sistema

\subsection{Descripción de las Piezas}

Como hemos comentado, el vector de características agrupa valores numéricos de características de cada pieza. En este caso, dichos valores numéricos deben ser convenientemente normalizados para evitar que influyan de modo desigual en el proceso de decisión. El vector consta de los siguientes datos:

- Área de la pieza

- Los 7 momentos de $\mathrm{Hu}$

Los momentos de Hu [Sonka, 93] son momentos invariantes a traslaciones, rotaciones y cambio de escala, que pueden calcularse a partir de los momentos centrales normalizados de orden 2 y 3 utilizando la ecuación (3). 

$\overline{\mathrm{VC}}$ que une el vértice de referencia $\stackrel{p}{\mathrm{~V}}_{\mathrm{r}}$ con el centro de masas $\stackrel{\rho}{\mathrm{C}}$. En segundo lugar calcularemos el eje de inercia $\mathrm{E}_{\mathrm{i}}$ del modelo geométrico. Del segmento $\overline{\mathrm{VC}}$ nos interesará determinar el ángulo $\alpha$ que forma con el eje de inercia $\mathrm{E}_{\mathrm{i}} \mathrm{y}$ su longitud $d$.

Una vez determinado el par $(\alpha, d)$ para la pieza prototipo, estamos en condiciones de identificar el vértice de referencia en la pieza percibida. Los pasos que tendremos que dar sobre la imagen de la pieza son:

- Calcular el centro de masas. 
- Calcular el eje de inercia.

- Utilizando el par $(\alpha, d)$ sobre el eje de inercia, obtener los dos puntos de la imagen candidatos a vértice de referencia.

- En estos dos puntos iniciaremos una búsqueda de vértice que deberá tener éxito en uno de los dos casos. Si tenemos éxito en los dos casos significa que la pieza es simétrica, no importando entonces

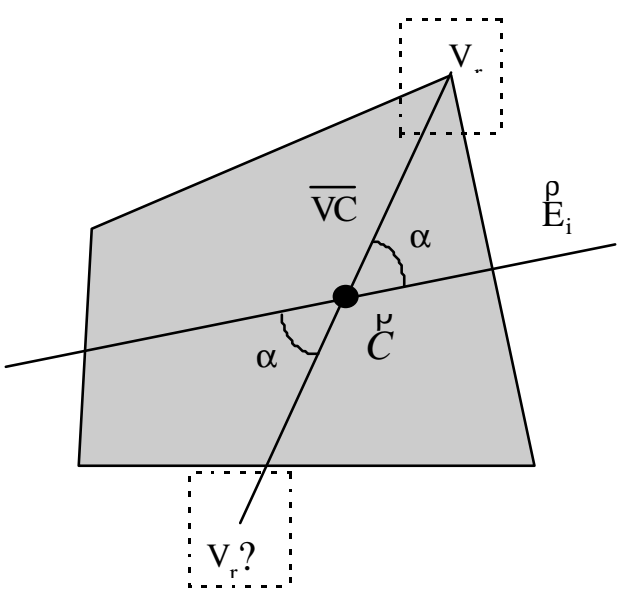

Figura 4: Determinación de la posición del vértice de referencia

\subsection{Búsqueda del Vértice de Referencia}

Como resultado del proceso anterior se ha obtenido una primera aproximación (dada la baja resolución proporcionada por la cámara lejana) a la posición del vértice de referencia. Para obtener un cálculo mucho más preciso, (a) desplazaremos la cámara cercana a la posición aproximada, (b) tomaremos una imagen con la cámara cercana y (c) procesaremos esta imagen para obtener con precisión la posición del vértice. El

- Se realizará un proceso morfológico de apertura [Dougherty, 92] para eliminar los agujeros que pudieran aparecer debido a la naturaleza del material.

- Se efectuará un proceso de barrido de la imagen para determinar las coordenadas de $2 \mathrm{~N}$ puntos de la frontera (borde) de la pieza, N puntos por cada lado. Sin entrar en detalles, existen 12 posibles tipos de vértice, que determinan el proceso de barrido a aplicar.

- Con estos dos conjuntos de $\mathrm{N}$ puntos determinaremos dos rectas de regresión que aproximarán los lados que cortan en el vértice de referencia.

- Calculando el punto de corte de las rectas de regresión obtenemos la posición del vértice de referencia.

\section{Posicionamiento Final de la Pieza}

Una vez conocidos el vértice de referencia y el eje de mínima inercia se puede calcular con la precisión requerida el lugar en el que se encuentra el centro de masas de la pieza. A ese lugar se enviará el efector del robot para, después de una traslación en su eje vertical con objeto de aprisionar la pieza contra la mesa, efectuar los movimientos (rotación y traslación) que la llevarán desde su posición inicial hasta la final.

Estos movimientos, tanto los de acercamiento como los de posicionamiento, se realizan en base a los sistemas de referencia definidos en la caracterización geométrica del entorno y utilizando las expresiones matriciales dadas en la ecuaciones 1 y 2 . Este proceso se realiza en los siguientes pasos: 
- transformación de las coordenadas del centro de masas referidas al sistema de referencia $\mathrm{R}_{\mathrm{C}}$ de la cámara cercana al sistema de referencia del mundo,

- cálculo de las coordenadas articulares finales que describen el movimiento del robot hasta ese punto, en el sistema de referencia del mundo,

- realización de la rotación y traslación de la pieza hasta la posición final, en el sistema de referencia del mundo, y

- liberación de la pieza y retirada del efector de la zona de trabajo.

\section{Conclusiones}

Es este artículo hemos presentado el proyecto FAMA (Fabricación Avanzada de Material Aeronáutico), un prototipo que incluye todas las fases clásicas [Fu, 89] de un sistema de visión artificial. Su mayor interés radica en la utilidad, versatilidad y funcionamiento en tiempo real (a una tasa de unas 3 piezas por minuto) y en la integración con el control de un brazo robótico de tipo pórtico.

Hemos proporcionado la definición de un proceso de producción adaptado al problema, en el que engarzan elementos de análisis de imágenes con elementos de control del brazo, permitiendo la utilización de un sistema doble de cámaras que, abaratando costes, proporciona una precisión equivalente a la de una cámara de alta

Del mismo modo, se ha caracterizado la geometría del sistema integrado brazo/cámara-cercana/cámaralejana y obtenido sus parámetros de Denavit-Hartenberg, lo que permite la modelización del entorno de operación del robot. Así mismo, la parametrización del sistema hace posible el ajuste por calibración de los aspectos variables de los elementos constitutivos, tales como la orientación y altura de las cámaras.

Los procedimientos de clasificación utilizados proporcionan un rendimiento óptimo para el conjunto de piezas a tratar. No obstante, gracias a su diseño modular, el sistema puede ser fácilmente modificado o ampliado para incorporar nuevas características o procesos de clasificación más complejos. En lo referente al posicionamiento, los errores obtenidos están por debajo de los requisitos iniciales del proyecto.

Las técnicas de coordinación de varias cámaras para conseguir un objetivo común empleadas en este proyecto podrían ser utilizadas en otros de características similares, tales como la clasificación de documentos, inspección visual de defectos de fabricación, vigilancia en sistemas de seguridad, etc.

\section{Referencias}

[Denavit, 55] J. Denavit, R.S. Hartenberg. "A Kinematic Notation for Lower-Pair Mechanics based on Matrices”, ASME J. Applied Mechanics, June 1955, pp. 215-221.

[Dougherty, 92] R.E. Dougherty. An Introduction to Morphological Image Processing. SPIE-The International Society for Optical Engineering, 1992.

[Fu, 89] K.S. Fu, R.C. González; C.S.G. Lee. Robotics: Control, Sensing, Vision, and Intelligence. McGraw-Hill, New York, 1989.

[Maravall, 93] D. Maravall. Reconocimiento de Formas y Visión Artificial. Ra-Ma, Madrid, 1993.

[Pratt, 91] W.K. Pratt. Digital Image Processing. John Wiley \& Sons, New York, 1991.

[Schilling, 90] R.J. Schilling. Fundamentals of Robotics. Analysis and Control. Prentice Hall, New Jersey, 1990.

[Sonka, 93] M. Sonka, V. Hlavac, R. Boyle. Image Processing, Analysis and Machine Vision. Chapman \& Hall, 1993. 